\title{
AirExGlove - A Novel Pneumatic Exoskeleton Glove for Adaptive Hand Rehabilitation in Post-Stroke Patients
}

\author{
Agostino Stilli, Arianna Cremoni, Matteo Bianchi, Alessandro Ridolfi, Filippo Gerli, \\ Federica Vannetti, Helge A. Wurdemann, Benedetto Allotta and Kaspar Althoefer
}

\begin{abstract}
Stroke is one of the leading causes of disability worldwide: post-stroke disabilities affect the upper and lower limbs, significantly undermining a subject's autonomy in the Activities of Daily Living (ADLs). Among post-stroke disabilities, one of the most impairing and widespread conditions is the clenched fist deformity: the subject experiences a permanent contraction of the hand, resulting in a closed hand rest pose. In this paper, the authors propose a novel light-weight inflatable soft exoskeleton device, called the AirExGlove, to deliver high-dosage, adaptive and gradual rehabilitation therapy to patients affected by clenched fist deformity. Our system is lightweight, low-cost, adaptable to any hand size and unobtrusive. The system has been extensively tested to assess the hand-opening range in which it can operate according to the severity of the patient condition, which is typically ranked on the Modified Ashworth Scale (MAS) scale. Experimental analysis demonstrates the suitability of the glove for patients affected by post-stroke muscle spasticity scoring up to 3 out of 4 in the MAS scale. Preliminary testing with clenched-fist patient confirmed a higher level of ergonomics of the system in comparison with rigid-linked robotic systems.
\end{abstract}

\section{INTRODUCTION}

Despite the advancements in modern medicine, stroke is still one of the leading causes of disability worldwide [1]. The hospitalization, rehabilitation and long-term care of post-stroke patients account for a considerable amount of human and financial resources usually born by the healthcare system, affecting transversally high [2], middle and low income countries [3]. People who survive a stroke are often left with long-term problems due to motion disabilities in the limbs. One of the most diffused condition among stroke survivors is spastic hemiplegia, an invalidating neuromuscular condition of spasticity. Subjects experience a constant contraction in the muscles on one side of the body, due to the nerve damage caused by the stroke in the opposite brain hemisphere. Such condition frequently results in clenched fist deformity.

\footnotetext{
* The research has received funding from the European Commission's

Horizon 2020 Research and Innovation Programme, project FourByThree under grant agreement No 637095.

A. Stilli, is with the Department of Informatics, King's College London, Strand, London WC2R 2LS, UK (e-mail: agostino.stilli@kcl.ac.uk)

A. Cremoni, M. Bianchi, A. Ridolfi and B. Allotta are with the Department of Industrial Engineering (DIEF), Univ. of Florence, Via di S. Marta 3, Florence, Italy.

F. Gerli, F. Vannetti and B. Allotta are with Don Carlo Gnocchi Foundation, Via di Scandicci 269, 50143, Florence, Italy.

H. Wurdemann is with the Department of Mechanical Engineering, Univ. College London, Gower St, London WC1E 6BT, UK

K. Althoefer is with the School of Electronic Engineering \& Computer Science, Queen Mary Univ. of London, Mile End Rd, London E1 4NS, UK.
}

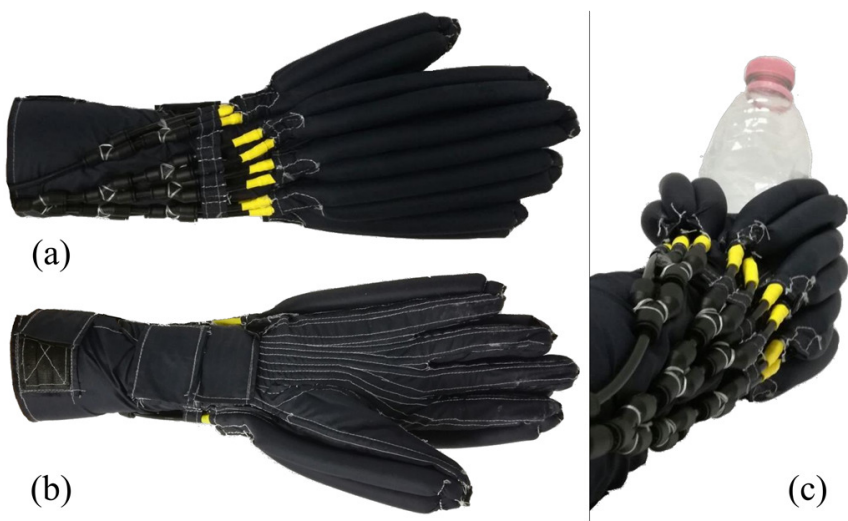

Figure 1 - Overview of the AirExGlove: (a) hand dorsal side of the system (pneumatic chambers pressurized), (b) hand palm side (tendon channel seams visible in white) and (c) side view of the system (thumb side) when grasping a plastic bottle.

Hand(s) (one in case of hemiplegia, both in case of diplegia) are always contracted, impairing subjects during hand-opening tasks. A number of robotic devices have been developed for hand-rehabilitation in the past decades [4]. Both exoskeletons and end-effector devices can provide additional support during physiotherapy [5]. The effectiveness of robotic-assisted therapy has shown significant impact in a number of studies [6]. In particular, these systems have outperformed standard rehabilitation routines in case of upper limb extremities [7] resulting in the growing development of hand rehabilitation devices. The ergonomics of hand exoskeletons has been significantly improved by soft robotics: a number of systems have been developed using soft materials such as elastomers [8], [9] and fabric [10], [11], making use of pneumatic and tendon actuation.

The use of Bowden cables has been also explored for soft systems [12]. However, the majority of systems based on elastomers provide only a single direction actuation: e.g. the systems presented in [9], [13] can only assist when closing but not when opening the hand. Intermediate opening configurations can only be achieved by a challenging interplay between a certain pressure value which is opposed by the muscle activity of the fingers. These exoskeletons are usually pneumatically actuated with the actuation system being mounted on the back of the hand, because if mounted on the palm the system would represent an obstacle to most manipulation tasks, due to the encumbrance of these actuators. Systems based on fabric structures using tendon actuation such as those presented in [10], [11], [14] can support both closure and opening of the hand. 


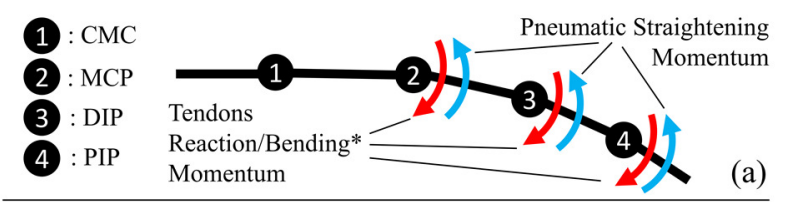

$\Rightarrow$ Air Pressure Force $\Rightarrow$ Sleeve Reaction Force $\Rightarrow$ Tendon Reaction/Pulling* Force

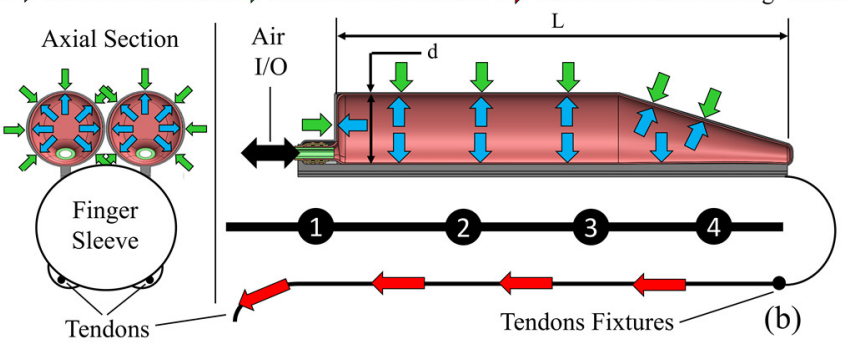

Figure 2 - AirExGlove actuation principle: (a) a schematic of the long fingers is depicted, showing the abbreviation of the joints names: metacarpo-phalangeal (MCP), proximal interphalangeal (PIP) and distal interphalangeal (DIP). Inside the palm, each finger is connected to a metacarpal bone through the carpo-metacarpal joint (CMC). The momenta provided by the pneumatic (azure curved arrows) and tendon driven (red curved arrows) actuation system are also depicted. (b) From left to right, a radial and a longitudinal section view of a single chamber are shown. Different arrows are used to illustrate the distribution of the air pressure forces (azure), the reaction forces produced by the sleeve (green), the pulling forces exerted by the tendons (red). The double-headed arrow indicates the input/output channel for the pressurized air. Geometrical parameters of the chambers d (diameter) and L (length) are also indicated. *Reaction momentum when the tendon actuation is used to limit the opening movement (rehabilitation therapy), bending momentum when it is used to simulate the clenched fist condition (Simulations of muscle spasticity, see Section III)

Due to the low diameter of the used actuation means (here: the tendons) these can easily be guided along both sides of the hand. Both tendons and pneumatic actuators have been used to assist in hand-opening and hand-closing tasks. On one hand, tendon actuation is considered light weight, but can exerts undesired longitudinal forces to the fingers and joints. On the other hand, pneumatic soft actuators are generally bulky, but provide the desired opening/closure force with marginal longitudinal forces being applied on the fingers.

In the context of the rehabilitation of post-stroke patients affected by clenched-fist deformity, a solution based on a fabric structure and a plastic actuator has been proposed in [15]. However, similar to the hand-closing pneumatic actuators presented in [9], achieving intermediate stable finger positions is challenging due to the single pneumatic actuation mechanism proposed. Also, the achieved stability of the opening configuration depends on the equilibrium between the closure force exerted by the patient and the opening force provided by the actuators, resulting in unstable control of the opening movement.

In this paper, we introduce a novel inflatable exoskeleton glove called AirExGlove. Our glove makes use of an antagonistic actuation principle (introduced in [16]-[18]) which is inspired by creatures in nature that are able to activate opposing sets of muscles to contextually control the pose and the stiffness of natural limbs. To the best of our knowledge, this is the first time, a hybrid solution as proposed in this paper is applied to an exoskeleton glove combining the benefits of both pneumatic and tendon-driven actuation to deliver gradual rehabilitation therapy to patients with hand-opening disabilities, such as clenched fist in poststroke patients: pneumatic actuators are located on the back of the hand to assist the user during hand-opening tasks. Tendons are guided along the palm of the device and are used to mechanically limit the opening movement to the desired level, providing a shape constraint. With the proposed system, a stable equilibrium is achieved between the opening force provided by the pneumatic actuators and the reaction force provided by the tendons, independently from the closure force applied by the patient. As a result, any intermediate configuration between a fully open and closed hand can be achieved.

This paper is organized as follows: the design and the control system of the AirExGlove are described in Section II. The experimental setup and results are presented in Section III. Section IV presents the conclusions and suggests possible improvements and other potential applications.

\section{MATERIALS AND METHODS}

Our presented exoskeleton is intrinsically and extrinsically actuated: the pneumatic actuation system, which provides the straightening momentum and, hence, supports the opening movement of the hand, is mounted on the back of the hand (intrinsically) whereas the tendon-driven system, which mechanically limits the opening movement to the desired aperture, is located inside the hand palm (extrinsically), Figure 1 (a) and (b), respectively.

In the design process, we aimed our novel AirExGlove:

- to deliver gradual rehabilitation therapy to patients affected by hand-opening disabilities.

- to be portable and lightweight, using fabric material for the exoskeleton structure and a small number of plastic components, the system is lightweight and portable.

In addition to these design features, our system has been conceived to be unobtrusive and scalable - the fabric structure is based on standardized glove shapes and sewing templates. Hence, its design can be easily adjusted to fit any hand size. The pressure level in the pneumatic actuators, hence, the straightening momentum exerted on the fingers, can be adapted according to the severity of the spasticity condition of the subject. It is also of paramount importance for the patient to make use of the entire palm without being obstructed by the assistive technology since in the palm most manipulation with the environment occurs. The thickness of the AirExGlove palm is comparable to a thin glove; on the dorsal side, the thickness of our system is less than the diameter of a finger (Figure 2 (b), d=13.5 mm for the tested system). Commercially available nylon fabric, sewing threads, long balloons and other small components such as rubber pipes, cable ties, heat-shrink tubes and pipe connectors have been used to create this cost-effective and low-cost system (total value of components is less than $£ 10$ ). The proposed system is based on the work firstly proposed by the authors in [19] and builds on the work presented in [17], [18]. The AirExGlove aims at the rehabilitation and assistance for patients affected by clenched fist deformity in performing the Activities of Daily Living (ADLs). 


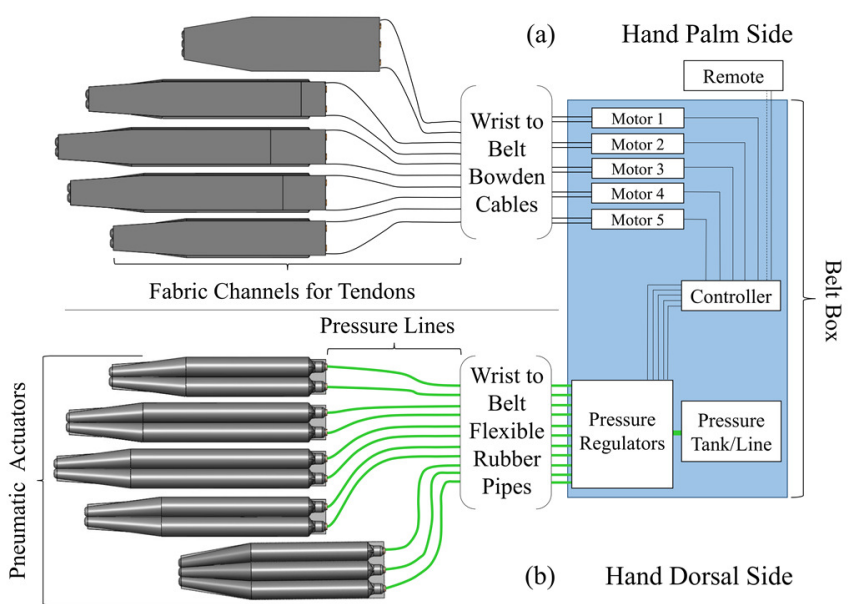

Figure 3 - AirExGlove chambers arrangement and intended control scheme: the system comprises independent actuation for each finger (two chambers for the long fingers, three chambers for the thumb and two tendons for each finger). The pressure regulators, the motors to actuate the tendons, the controller, the pressure tank (or the connection to the pressure line) and the battery are embedded in a box on a belt to be worn by the patient. Tendons are routed using Bowden cables from the belt to the wrist, hard rubber pipes are used for the routing of the pressure lines.

The soft materials used guarantee high ergonomics and an inherently safe interaction between the user and the device, as well as between the user and the external environment. The device is also safe during physical interactions with other individuals.

\section{A. Working Principle, Design and Materials}

Figure 1 shows a prototype of the AirExGlove composed of four main elements: a customized fabric glove made of coated Nylon PU (55 g/m², ukfabricsonline.com), an inclusive wristband with an adjustable Velcro closure, a set of pneumatic actuators (mounted on the hand's dorsal side) and a set of tendons (fixed on the palm). The palm of the glove presents a double fabric layer allowing the routing of the tendons guided along two parallel seam lines for each tendon channel. A professional sewing machine (PFAFF Creative 3.0) has been used to create the prototype. The fabric used for the glove is flexible but not stretchable and, thus, provides a good force transmission between the exoskeleton and the hand without reducing dexterity. Inextensibility of the fabric is also a critical requirement for the embedded actuators to ensure the required forces and stiffness. The glove weighs $90 \mathrm{~g}$.

The working principle and the design of the system for one of the long fingers are shown in Figure 2 (a) and Figure 2 (b). A schematic of a long finger showing the bone/joint chain is also depicted in Figure 2 (a). Abbreviations are listed in Figure 2 (a) next to the respective joint numbers. Each chamber of the pneumatic actuators is composed of an airtight latex bladder pressurised through an input/output channel (see Figure 2 (b) Air I/O), fitted inside a fabric sleeve. Seam lines along the tendon channels are also visible in Figure 2 (b). Once a positive air pressure is applied to the bladder, the chamber inflates. The fabric sleeve - due to its inextensibility - limits the inflation to a predefined cylindrical shape as shown by the axial section in Figure 2 (b). The balance between the pressure force of the air (azure coloured arrows in Figure 2 (b)) and the reaction force of the fabric sleeve (green coloured arrows in Figure 2 (b)) generates the straightening momentum shown by the azure coloured curved arrows in Figure 2 (a). It is important to note that, differently from the system presented in [15], the chambers of our system are not expanding against the dorsal side of the glove, hence, no compression force is perceived by the user, assuring his/her comfort. As shown in Figure 1 the pneumatic actuators cover the whole length of the fingers and their respective metacarpal section. The straightening momentum indicated in Figure 2 (a) tends to align each finger group of three (two for the thumb) phalanxes with the respective metacarpal bone. On the other hand, controlling the tendon displacement, we can limit the opening movement to the desired amount providing a reaction momentum that opposes the one generated by the pneumatic actuators, once such threshold is reached. The equilibrium between these two momenta allows stable positioning of the fingers in any intermediate configurations between full hand opening to the full closure. Each finger is actuated by a pair of tendons both fixed at the fingertip in Figure 2 (b). Each pair of tendons is controlled by the same actuator; hence they experience the same displacement. The tendons are routed on the side of the finger pads; thus, they hardly interfere during grasping tasks. The design of the glove allows high customizability both in regards of the hand size and the forces exerted on the fingers by the pneumatic actuation. Tailored gloves can be manufactured according to the user's hand shape and by modularity of the pneumatic actuators the number and dimension of the chambers can be defined based on the user needs and pre-existing medical conditions.

\section{B. Control system and actuators arrangement}

In Figure 3 the envisaged control system for the AirExGlove is presented. Actuators are located in a box fixed on a belt, tendons are routed using Bowden cables along the back, the shoulder and the arm up to the user wrist. Same applies to the flexible rubber pipes used to supply pressurised air to the pneumatic actuators. The weight of the envisioned actuation system is estimated to be less than $3 \mathrm{~kg}$ for the configuration shown in Figure 3 (five motors/five pressure regulators) and less than $2 \mathrm{~kg}$ for an underactuated configuration with only one motor to pull all the tendons together and one pressure regulator for all the chambers, satisfying the weight requirements for a backpack/belt solution for the actuators as indicated in [20].

As illustrated in Figure 3 and Figure 1, the system comprises of five pneumatic actuators. Each of the four actuators on the hand dorsal side of the fingers is composed of two pneumatic chambers. The thumb has a higher grasping-force-to-length ratio; hence, we make use of three rather than two chambers to keep the same dorsal encumbrance. Applying this antagonistic actuation principle, we can not only control the maximum finger opening generated by the pneumatic actuators (thus, deliver gradual rehabilitation therapy to patients with hand opening disabilities), but also use the same system to assist patients with different pathologies during hand closure tasks. 


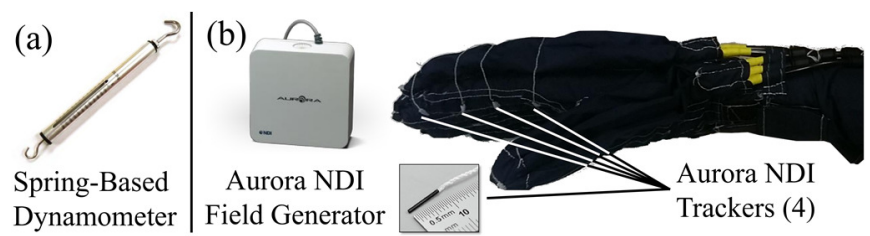

Figure 4 - Experimental setup for tendon pre-loading tests: (a) the dynamometer used for tendon tension measurements. (b) Magnetic trackers and field generator Aurora NDI system for fingertip, PIP, DIP and MCP trajectory data acquisition.

\section{IRB Statement}

The tests described in Section III (A to C) were conducted at King's College London in London, UK. During these tests, the investigated system was solely worn by Arianna Cremoni, co-author of this work, a healthy subject with no impairment to the mobility of the hand. Ethical approval has been obtained by the Board of Ethics of the hosting institution for these tests, i.e. King's College London. (Study Title: Experimental evaluation of the grasping performance of a novel soft exoskeleton for human hand rehabilitation and empowering, KCL Ethics Ref: LRS-16/17-3631.)

The tests described in Section III.D were conducted at the Rehabilitation Centre "Don Carlo Gnocchi" in Florence, Italy. During these tests, the investigated system was solely worn by a male patient, 54 y/o affected by spinal muscular atrophy type 2 . The tests were conducted under the supervision of Mr. Gerli and Dr. Vannetti, co-authors of this paper. Informed consent and information sheet for the subject are available at: http://bit.ly/2oyP92e; According to the policy of the Rehabilitation Centre IRCCS "Don Carlo Gnocchi", ethical approval is not required for the one-time qualitative tests conducted. Written confirmation has been also obtained from the director of the centre, Prof. Carlo Macchi. This document is available at: http://bit.ly/2oyP92e.

\section{EXPERIMENTAL SETUP AND RESULTS}

The level of hand muscle spasticity of post-stroke patients can be clinically assessed by trained physicians/physiotherapists by manually stretching the fingers of the subject in the whole range of motion of the hand, thus evaluating the level of contraction experienced by assigning a score from 0 to 4 on the Ashworth Scale. The Ashworth Scale, firstly introduced in [21] and later extended in [22] in the Modified Ashworth Scale (MAS), has been proven to be a reliable method for this assessment [23]. The scale assigns numerical values from 0 to 4 to grade the level of spasticity measured, where 0 reflects no increase in muscle tone, 1/1+ a slight increase, 2 a more marked increase, but affected part(s) easily moved, 3 a considerable increase, passive movement difficult and 4 that the affected part(s) are rigid in flexion or extension [22]. A set of experiments has been conducted to evaluate the performance of the AirExGlove. These tests involved a healthy subject (female, 25, $171 \mathrm{~mm}$ wrist-to-index-finger-tip length, $75 \mathrm{~mm}$ palm width, hand size $S$ ). The embedded tendon system was utilised to simulate the muscle spasticity, applying a known load to the tendons (bending momentum applied by the tendons, see Figure 2:*).
Independent measurements were performed for all the four fingers, but given the high degree of similarity of the data collected, only the data of the index have been included

The presented study includes four experimental sets:

A. Tendon pre-loading for clenched fist simulation Hand contractions experienced by patients who are affected by clenched fist deformity are simulated. We apply different loads on the tendons and then clinically assess the equivalent MAS score of the system. During these tests, the pneumatic actuators were not pressurized.

B. Workspace analysis with no pre-load on the tendons The workspace of the AirExGlove when the user has a healthy hand is evaluated (MAS Score $=0$, pneumatic actuators pressurized in the range $0 \mathrm{kPa}-300 \mathrm{kPa}$ )

C. Workspace analysis with pre-loaded tendons We investigate the performance of the exoskeleton when the user is affected by clenched fist deformity simulating this condition applying a pre-load on the tendons (MAS Score $\geq 1$, pneumatic actuators pressurized in the range 0 $\mathrm{kPa}-300 \mathrm{kPa})$.

D. Preliminary testing with clenched-fist patient. We conducted a preliminary user test on a single patient with clenched fist deformity to evaluate the performance and the ergonomics of the system.

\section{A. Tendon pre-loading for clenched fist simulation}

To simulate the contraction force typical of clenched fist deformity, we actuate all tendon pairs. The tendon pair of the index finger is loaded using the spring-based dynamometer ( 0 $-10 \mathrm{~N}$ scale, spring constant $\mathrm{k}=100 \mathrm{~N} / \mathrm{m}$ ) shown in Figure 4 Figure 4(a). The loading procedure is as follow: the top eyelet of the instrument was connected to a hook bonded to a linear module, the movable bottom eyelet of the instrument was connected to the tendon pair. The dynamometer was then moved so to achieve the desired tension on the tendon pair, once the desired pre-load was achieved the linear module was fixed in position. To clinically assess the (equivalent) MAS score of the AirExGlove when a load is applied on the tendons to simulate a clenched fist deformity, a trained physiotherapist, one of the authors, Mr. Gerli, from the "Don Carlo Gnocchi" Research Institute, in Florence, Italy has been involved. The procedure for the MAS score assessment has been detailed in the Introduction of this section. The use of a spring-based dynamometer (rather than a load-cell one) is critical for this test, because it provides the needed compliance when the fingers are manually extended. The results are reported in Table 1 . As the data show the force range $0 \mathrm{~N}-5 \mathrm{~N}$ is enough to simulate the MAS range $0-3$.

Table 1 - Tendon pre-load $F_{\text {Preload }}[\mathrm{N}]$ to MAS Score mapping.

\begin{tabular}{|c|c|c|c|c|c|c|c|c|c|c|c|}
\hline$F_{\text {Tendons }}[\mathrm{N}]$ & 0.25 & 0.5 & 0.75 & 1 & 1.25 & 1.5 & 1.75 & 2 & 2.25 & 2.75 & $3-5$ \\
\hline MAS Score & 1 & 1 & $1+$ & 2 & 2 & 2 & 2 & 2 & $2+$ & $2+$ & 3 \\
\hline
\end{tabular}

\section{B. Workspace analysis with no pre-load on the tendons}

In this section, we evaluate the workspace of the system when no pre-load is applied on the tendons, i.e. when used by a patient with no spasticity. In the first phase of this set of experiments, the subject is asked to perform a complete hand opening to provide a reference trajectory. Measurements are obtained with the 3D magnetic tracking system (Aurora 
NDI) shown in Figure 4 (b) (markers in correspondence of MCP, DIP, PIP and the fingertip; base reference measured on the CMC). In the second phase, the subject is asked to close the hand. The system is then pressurised applying 100 $\mathrm{kPa}, 200 \mathrm{kPa}$ and $300 \mathrm{kPa}$ of pressure. The subject is asked not to intentionally resist neither assist the system during the movement. Tendons are kept loose during these tests The processed data by the magnetic tracker system during the four tests are presented in Figure 5. The trajectories described by the fingertip and the joints in the pressure actuated case follow those in the free hand-opening case, providing a natural movement to the user. Figure 6 shows the maximum diameter of graspable objects for different pressure levels; the circumferences shown are the largest for which each phalanx is tangent, hence, in contact, with the grasped object in the three cases. The data show that the AirExGlove allows a hand opening sufficient for most of the common grasping tasks, e.g. grasping a glass $(70 \mathrm{~mm}-90$ $\mathrm{mm} \varnothing)$ or a bottle $(90 \mathrm{~mm}-100 \mathrm{~mm} \varnothing)$ even at low pressure.

\section{Workspace analysis with pre-loaded tendons}

In this section, we present the data related to the workspace analysis of the system when a pre-load is applied on the tendons: these scenarios are equivalent to common hand contractions of patients affected by clenched fist deformity. In this set of experiments, we are simultaneously measuring the force on the tendons, the pressure in the pneumatic chambers and the pose of the finger. Also here we present the data for the index finger.

The experiments are conducted as follows: 1 - the subject is requested to close and then relax the hand, $2-$ a pre-load is imposed on the index tendon pair (using the same spring-based dynamometer used during the clinical assessment of the system) inducing a certain closure level on the hand, 3 - the pneumatic actuator is gradually pressurised up to $300 \mathrm{kPa}$ inducing the desired opening movement, consequently increasing the tension on the tendon pair. This procedure is repeated for each pre-load level from 1-5 N. According to the data of the first experimental set, the system exerts a normal force on the finger pad in the range of 3-5 N. Figure 7 shows the dependence between the pressure applied to the pneumatic actuators and the force measured on the tendon pair. Each curve corresponds to a different pre-load condition (1-5 $\mathrm{N}$ range, $1 \mathrm{~N}$ steps). Pressure is measured and increased by steps of $33 \mathrm{kPa}$ in the range of $0-100 \mathrm{kPa}$ and steps of $20 \mathrm{kPa}$ in the range of 1-300 $\mathrm{kPa}$. A linear approximation is shown for each data set in Figure 7 (a). To quantify the opening movement, a percentage is calculated based on the opening movement in the free hand-opening case. These values are included for all discrete points in Figure 7. The dependence between the opening percentage values, the force acting on the tendons and the pressure inside the pneumatic actuators is shown for the pre-load range 1-5 $\mathrm{N}$ in Figure 7 (b). Hence, it is possible to define the requested pressure and achieve a desired opening movement for a patient experiencing a certain contraction on the hand. These data refer to our subject (female, hand S size). Similar curves can be derived for any hand size.
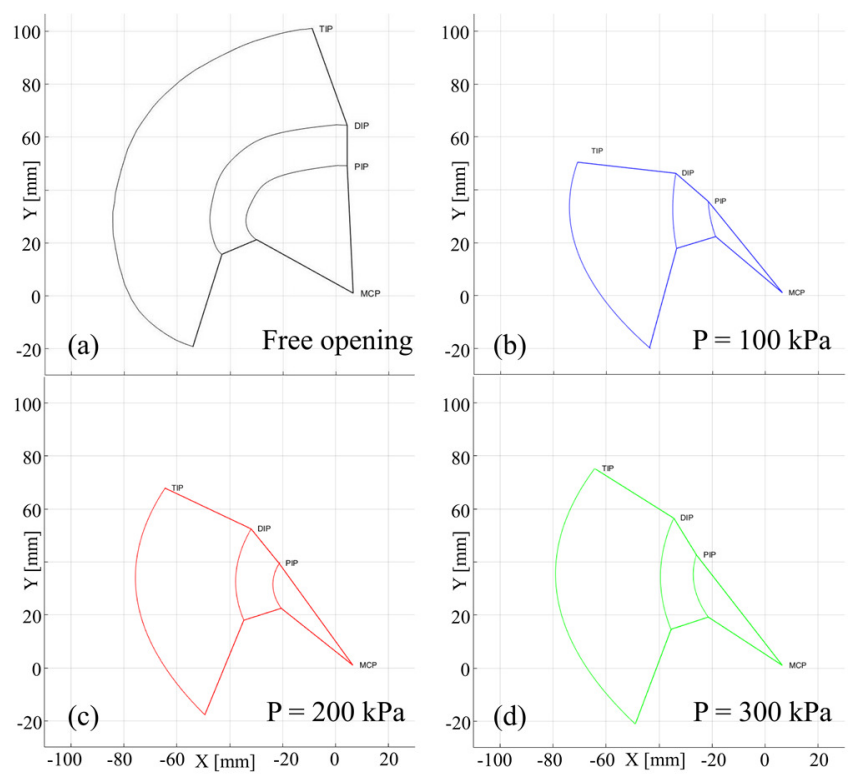

Figure 5 - Trajectories of the index in case of free opening (a) and when different pressures are applied to the pneumatic actuators: $100 \mathrm{kPa}(\mathrm{b}), 200$ $\mathrm{kPa}$ (c) and $300 \mathrm{kPa}$ (d). Tendons are kept loose (no pre-load). Data collected using the Aurora NDI tracking system with markers on the MCP, DIP, PIP and fingertip.

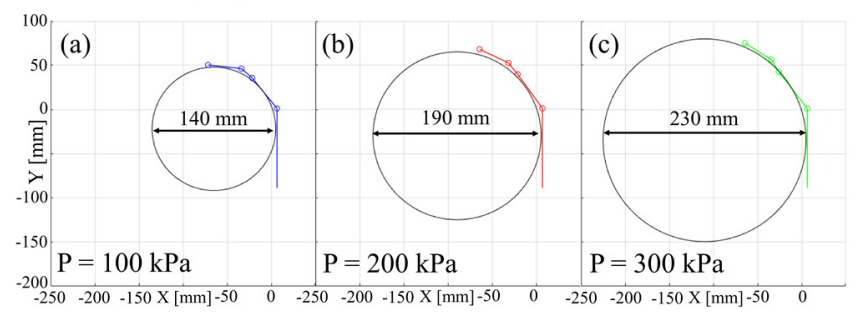

Figure 6 - Maximum diameter of graspable objects with no pre-load on the tendons for different pressure levels.
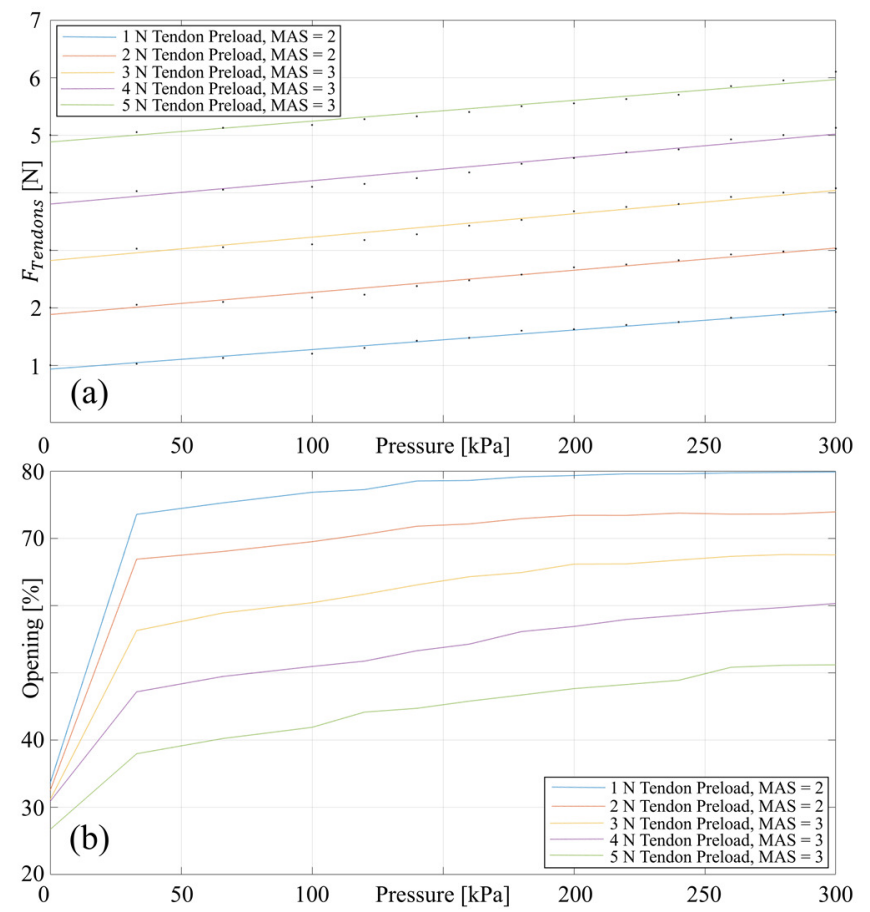

Figure 7 - Index finger system characterization for hand opening using pneumatic actuators with tendon pre-load applied (clenched fist simulation): (a) pre-load on the tendons $F_{\text {Preload }}[\mathrm{N}]$ and (b) opening percentage [\%] for different pre-loads, in the pressure range 0 to $300 \mathrm{kPa}$. 
The system was able to provide an opening up to $79 \%$ for $1 \mathrm{~N}$ of pre-load (MAS =2) and up to $51 \%$ for $5 \mathrm{~N}$ of preload (MAS $=3$ ) at $300 \mathrm{kPa}$.

\section{Preliminary testing with clenched-fist patient}

Non-quantitative tests of the system with a subject with clenched fist deformity (male, $54 \mathrm{y} / \mathrm{o}$, hand length: $17.5 \mathrm{~cm}$, hand width: $6 \mathrm{~cm}$, pathology: spinal muscular atrophy type 2, clenched fist deformity due to forearm tendons retroaction) with an equivalent MAS score of 1+ were successfully conducted at the Rehabilitation Centre "Don Carlo Gnocchi" in Florence, Italy, under the supervision of Mr. Gerli and Dr. Vannetti, authors of this paper. The results are presented in the video attachment to this work. Informed consent and information sheet for the subject are available at the following link: http://bit.ly/2oyP92e. The test subject is a long-term collaborator of the research institute, with extensive experience in testing new rehabilitation devices.

\section{CONCLUSIONS}

In this paper a novel soft, inflatable and flexible exoskeleton for hand rehabilitation in post-stroke patients has been presented. This system builds on our previous work where a fabric sleeve is used to limit the inflation of a latex bladder to create a stiffness-tunable robotic manipulator [19], [24], [25]. The same actuation principle is used to create a soft, customizable, ergonomic and low-cost system. The data collected suggest that the AirExGlove is suitable to deliver high-dosage rehabilitation therapy to patients affected by post-stroke muscle spasticity scoring up to 3 in the MAS.

Preliminary testing of the system has been conducted on a single patient with clenched fist deformity. The subject has given enthusiastic feedback about the system, particularly appreciating its ergonomics, natural movement, unobtrusiveness during interactions with the environment and lightness. The capabilities of the system of stably achieving any intermediate hand-opening movements between full hand opening and closure when worn by a subject affected by clenched fist deformity have been tested and confirmed.

In future work, we will consider optimising the balance between the applied pressure and the size of the air chambers, to minimize the thickness of the pneumatic actuators. A characterization for all hand sizes, male and female, will be carried out to develop a comprehensive mathematical model, accounting for the geometrical parameters of the system, the air pressure and the MAS score. Further investigation will also be conducted to evaluate the effect of the reaction forces at the joints of the hand digits to better assess the comfort experienced by the user. As suggested by the work presented in [26] these forces can be significant, hence, further investigation is required. The actuation system will be developed with the aim of optimizing system portability. A case of study with multiple patients will be carried out for future work, keeping the involvement of experts in the field of rehabilitation and physiotherapy.

\section{REFERENCES}

[1] H. Wang et al., "Global, regional, and national life expectancy, all-cause mortality, and cause-specific mortality for 249 causes of death, 1980-2015: a systematic analysis for the Global Burden of Disease Study 2015,' Lancet, vol. 388(10053), pp. 1459-1544, 2016.
[2] D. Epstein, A. Mason, and A. Manca, "The hospital costs of care for stroke in nine European countries," Health Econ., vol. 17(S1) pp. S21-S31, 2008.

[3] D. O. Abegunde, C. D. Mathers, T. Adam, M. Ortegon, and K. Strong, "The burden and costs of chronic diseases in low-income and middle-income countries," Lancet, vol. 370(9603), pp. 1929-1938, 2007.

[4] B. R. Brewer, S. K. McDowell, and L. C. Worthen-Chaudhari, "Poststroke Upper Extremity Rehabilitation: A Review of Robotic Systems and Clinical Results," Top. Stroke Rehabil., vol. 14(6), pp. 22-44, 2007.

[5] J. Mehrholz and M. Pohl, "Electromechanical-assisted gait training after stroke: a systematic review comparing end-effector and exoskeleton devices," J. Rehabil. Med., vol. 44(3), pp. 193-199, 2012.

[6] P. S. Lum, C. G. Burgar, P. C. Shor, M. Majmundar, and M. Van der Loos, "Robot-assisted movement training compared with conventional therapy techniques for the rehabilitation of upper-limb motor function after stroke," Arch. Phys. Med. Rehabil., vol. 83(7), pp. 952-959, 2002.

[7] W. H. Chang and Y.-H. Kim, "Robot-assisted therapy in stroke rehabilitation," J. stroke, vol. 15(3), p. 174, 2013.

[8] H. K. Yap, J. H. Lim, F. Nasrallah, F. Z. Low, J. C. H. Goh, and R. C. H. Yeow, "MRC-glove: A fMRI compatible soft robotic glove for hand rehabilitation application," IEEE Int. Conf. Rehabil. Robot., vol. 2015Septe(65), 2015, pp. 735-740.

[9] P. Polygerinos et al., "Towards a soft pneumatic glove for hand rehabilitation,” IEEE Int. Conf. Intell. Robot. Syst., 2013, pp. 1512-1517.

[10] M. A. Delph, S. A. Fischer, P. W. Gauthier, C. H. M. Luna, E. A. Clancy, and G. S. Fischer, "A soft robotic exomusculature glove with integrated sEMG sensing for hand rehabilitation," IEEE Int. Conf. Rehabil. Robot., 2013.

[11] H. In, B. B. Kang, M. Sin, and K. J. Cho, "Exo-Glove: A wearable robot for the hand with a soft tendon routing system," IEEE Robot. Autom. Mag., vol. 22(1), 2015.

[12] M. Xiloyannis, L. Cappello, D. B. Khanh, S.-C. Yen, and L. Masia, "Modelling and design of a synergy-based actuator for a tendon-driven soft robotic glove," in 6th IEEE Int. Conf. Biomed. Robot. Biomechatr. (BioRob), 2016, pp. 1213-1219.

[13] H. K. Yap, J. H. Lim, F. Nasrallah, J. C. H. Goh, and R. C. H. Yeow, "A soft exoskeleton for hand assistive and rehabilitation application using pneumatic actuators with variable stiffness," in IEEE Int. Conf.on Robot and Autom., 2015, vol. 2015-June, pp. 4967-4972.

[14] D. Popov, I. Gaponov, and J.-H. Ryu, "Portable Exoskeleton Glove With Soft Structure for Hand Assistance in Activities of Daily Living," IEEE/ASME Trans. Mechatronics, vol. 22(2), pp. 865-875, 2017.

[15] H. K. Yap, J. H. Lim, J. C. H. Goh, and C.-H. Yeow, "Design of a Soft Robotic Glove for Hand Rehabilitation of Stroke Patients with Clenched Fist Deformity using Inflatable Plastic Actuators," J. Med. Device., no. c, 2016.

[16] H. A. Wurdemann, A. Stilli, and K. Althoefer, "Lecture notes in computer science: An antagonistic actuation technique for simultaneous stiffness and position control," in Int. Conf. on Intell. Robot. and Appl., 2015, pp. 164 174.

[17] F. Maghooa, A. Stilli, Y. Noh, K. Althoefer, and H. A. Wurdemann, "Tendon and pressure actuation for a bio-inspired manipulator based on an antagonistic principle," IEEE Int. Conf.on Robot. and Autom., 2015, pp. 2556-2561.

[18] A. Shiva et al., "Tendon-Based Stiffening for a Pneumatically Actuated Soft Manipulator,” IEEE Robot. Autom. Lett., vol. 1(2), pp. 632-637, 2016.

[19] A. Stilli, H. A. Wurdemann, and K. Althoefer, "Shrinkable, stiffnesscontrollable soft manipulator based on a bio-inspired antagonistic actuation principle," in IEEE/RSJ Int. Conf. Intell. Robot. Syst., 2014, pp. 2476-2481.

[20] P. Polygerinos, Z. Wang, K. C. Galloway, R. J. Wood, and C. J. Walsh, "Soft robotic glove for combined assistance and at-home rehabilitation," Rob. Auton. Syst., vol. 73, pp. 135-143, 2015.

[21] B. ASHWORTH, "Preliminary trial of carisoprodol in multiple sclerosis.," Practitioner, vol. 192, pp. 540-542, 1964.

[22] R. W. Bohannon and M. B. Smith, "Interrater reliability of a modified Ashworth scale of muscle spasticity," Phys ther, vol. 67(2), pp. 206-207, 1987.

[23] J. M. Gregson, M. Leathley, A. P. Moore, A. K. Sharma, T. L. Smith, and C. L. Watkins, "Reliability of the Tone Assessment Scale and the modified Ashworth scale as clinical tools for assessing poststroke spasticity," Arch. Phys. Med. Rehabil.,vol. 80(9), pp. 1013-1016, 1999.

[24] A. Stilli, L. Grattarola, H. Feldmann, H. A. Wurdemann, and K. Althoefer, "Variable Stiffness Link (VSL): Toward inherently safe robotic manipulators," in IEEE Int. Conf.on Robot. and Autom., 2017, pp. 49714976.

[25] A. Stilli, H. A. Wurdemann, and K. Althoefer, "A Novel Concept for Safe, Stiffness-Controllable Robot Links," Soft robotics, 4(1), pp.16-22, 2016.

[26] H. In and K.-J. Cho, "Analysis of the forces on the finger joints by a jointless wearable robotic hand, SNU Exo-Glove," in Converging Clinic. and Engin. Resear. on Neurorehab., Springer, 2013, pp. 93-97. 